\section{Insuficiencia tricuspídea funcional y miocardiopatía hipertrófica apical. Estrategia diagnóstica y terapéutica a propósito de un caso clínico}

\section{Functional tricuspid regurgitation and apical hypertrophic cardiomyopathy. Diagnostic and therapeutic strategy regarding a clinical case}

Mujer de 71 años con antecedentes de fibrilación auricular permanente y miocardiopatía hipertrófica apical ( $\mathrm{MCH}-\mathrm{A})$ que acude al servicio de emergencias por disnea en reposo y edemas en miembros inferiores. Presentaba cifras de presión arterial de $88 / 45 \mathrm{mmHg}$, con frecuencia cardiaca de $160 \mathrm{lpm}$, saturación periférica de oxígeno basal del $90 \%$, ingurgitación yugular severa y estertores crepitantes en ambas bases pulmonares. Además, tenía edemas hasta el tercio proximal de ambas piernas y hepatomegalia de $3 \mathrm{~cm}$. En el electrocardiograma se observó fibrilación auricular con respuesta ventricular rápida, signos de hipertrofia ventricular izquierda y ondas T negativas simétricas difusas (fig. 1A). Se realizó dímero-D negativo y radiografía de tórax en la que se observó cardiomegalia a expensas de cavidades derechas y redistribución vascular (fig. 1B) Se realizó ecocardiograma transtorácico que mostró un ventrículo izquierdo con volumen diastólico reducido $\left(36 \mathrm{ml} / \mathrm{m}^{2}\right)$, hipertrofia severa apical con grosor de $26 \mathrm{~mm}$ sin gradiente dinámico, fracción de eyección de ventrículo izquierdo conservada, disfunción diastólica severa con tiempo de deceleración de $80 \mathrm{~ms}$ y cociente $E / E$ ' de 16 , insuficiencia mitral degenerativa grado III, dilatación biauricular severa, ventrículo derecho dilatado con función sistólica deprimida ligeramente ( $S$ ' de $10 \mathrm{~cm} / \mathrm{s}$ y TAPSE de $15 \mathrm{~mm}$ ), válvula tricuspídea sin afectación orgánica significativa con insuficiencia severa central por déficit de coaptación de velos y dilatación del anillo tricuspídeo (44 mm) (figs. 1C y D). Ingresó en planta de hospitalización y se instauró tratamiento con dosis bajas de betabloqueante, furosemida y espironolactona. Durante los siguientes días se consiguió el control de la respuesta ventricular de la frecuencia cardiaca con dosis progresivamente crecientes de bisoprolol, además de resolver los signos congestivos, si bien la función renal sufrió deterioro significativo (creatinina pico de $2,8 \mathrm{mg} / \mathrm{dl}$ ) con resolución progresiva posterior tras la disminución de la dosis de diurético. Una vez lograda la estabilidad clínica y hemodinámica se realizó resonancia magnética cardiaca observando $\mathrm{MCH}-\mathrm{A}$ sin hipertrofia del ventrículo derecho con grado moderado de fibrosis mesocárdica en el área hipertrofiada, confirmando la insuficiencia tricuspídea (IT) de grado severo e insuficiencia mitral moderada (fig. 2). Además, se realizó cateterismo cardiaco que mostró arterias coronarias sin lesiones, gasto cardiaco de $1,8 \mathrm{l} / \mathrm{m}$, presión de aurícula derecha de $2 \mathrm{mmHg}$, presiones de ventrículo derecho: sistólica $28 \mathrm{mmHg}$, diastólica $2 \mathrm{mmHg}$; presiones de arteria pulmonar: sistólica $28 \mathrm{mmHg}$, diastólica $7 \mathrm{mmHg}$, media $14 \mathrm{mmHg}$; presión capilar pulmonar $9 \mathrm{mmHg}$, resistencias vasculares pulmonares 2,7 unidades Wood, y presiones de ventrículo izquierdo: sistólica $91 \mathrm{mmHg}$ y diastólica $53 \mathrm{mmHg}$. También se objetivó una tendencia a igualación de presiones diastólicas entre ambos ventrículos con morfología en dip-plateu (fig. 1E) y, aunque no fue completamente concluyente por la depleción de volemia, sugirió fisiología restrictiva.

Aunque la $\mathrm{MCH}-\mathrm{A}$ condicionase una fisiología restrictiva no observamos hipertensión pulmonar significativa por lo que esta no era el mecanismo fundamental que causaba la IT. La dilatación del anillo con relación a un remodelado auricular por fibrilación auricular y disfunción diastólica parecía la etiología principal de esta valvulopatía. Tras valoración en sesión médico-quirúrgica, y dada la clínica de insuficiencia cardiaca derecha, la severidad de la IT con repercusión sobre el ventrículo derecho y la disfunción diastólica severa con bajo gasto cardiaco se decidió realizar anuloplastia tricuspídea, recambio valvular mitral por prótesis mitral y practicar además miectomía apical. Se realizó la cirugía con buena evolución en el postoperatorio inmediato.

En el caso presentado, la conjunción de la $\mathrm{MCH}-\mathrm{A}$ y la IT severa hizo que el manejo clínico de la paciente fuera difícil ya que las herramientas del tratamiento médico para ambas enfermedades son «antagónicas». Por un lado, el tratamiento deplectivo es la herramienta clave en la insuficiencia congestiva derecha debido a la IT, si bien en la miocardiopatía hipertrófica $(\mathrm{MCH})$ el uso de diurético ha de ser cuidadoso ya que puede comprometer el gasto cardiaco y provocar gradiente dinámico. Por otro lado, el empleo de digoxina puede estar indicado en el caso de IT severa con fibrilación auricular rápida y disfunción ventricular derecha pero no es un fármaco adecuado en paciente con esta miocardiopatía. La titulación progresiva de betabloqueantes debido a las cifras de presión arterial y el manejo cuidadoso de los diuréticos hicieron posible alcanzar la estabilidad clínica y hemodinámica previa a la cirugía cardiaca. Otros antiarrítmicos, como la amiodarona, pueden ser útiles en estos casos como tratamiento cronotrópico negativo.

La MCH-A es una forma relativamente infrecuente de miocardiopatía hipertrófica (2-3\% de los pacientes), a excepción de Japón, donde fue descrita, llegando al $20 \%$ de enfermos con esta miocardiopatía ${ }^{1,2}$. Caracterizada por la presencia de hipertrofia predominante a nivel del ápex cardiaco, la edad media de presentación clínica es la cuarta década de la vida y su curso clínico es benigno comparado con las otras formas de $\mathrm{MCH}$ con una mortalidad anual del $0,1 \%^{3}$. La MCH-A se transmite con herencia autosómica dominante y hay pocas mutaciones específicas que provocan esta forma de $\mathrm{MCH}$, siendo la mayoría de las veces producida por mutaciones sarcoméricas que pueden provocar otras formas de $\mathrm{MCH}$ junto con factores modificadores (hemodinámicos, locales, etc. $)^{4}$. La característica diagnóstica más típica es la presencia de ondas $\mathrm{T}$ negativas profundas en derivaciones precordiales del electrocardiograma. La comorbilidad más común en estos pacientes es la fibrilación auricular, como en nuestro caso. Además, se pueden observar alteraciones de la contractilidad o formación de aneurismas ${ }^{3}$. El tratamiento médico es similar al del resto de fenotipos de miocardiopatía hipertrófica, pero a diferencia de las formas no apicales, los pacientes con $\mathrm{MCH}-\mathrm{A}$ requieren menos frecuentemente un implante de desfibrilador en prevención primaria y no se benefician del tratamiento invasivo para la obstrucción dinámica en el tracto de salida al no estar presente en ellos. 

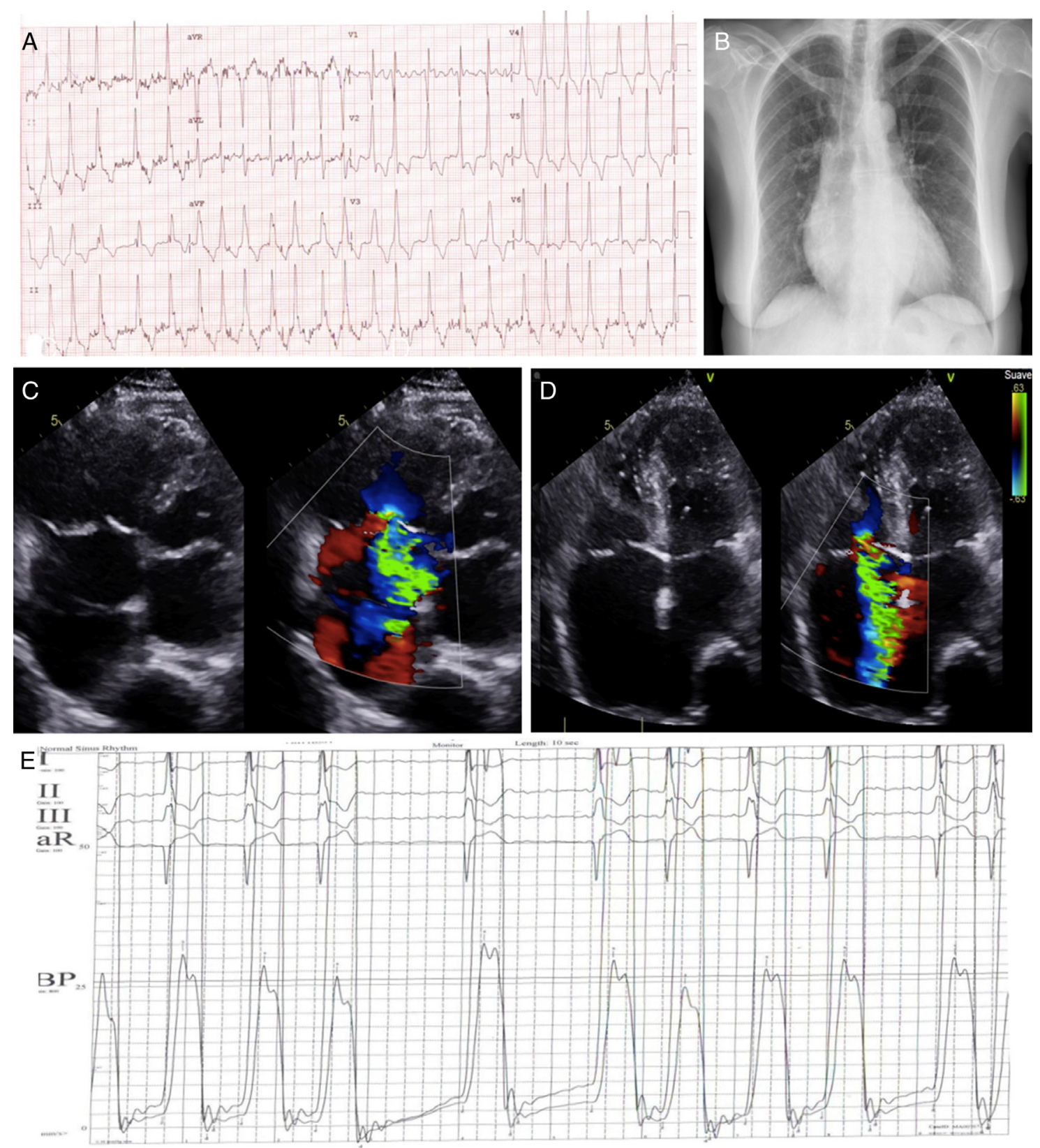

Figura 1 A) Electrocardiograma de la paciente en urgencias que muestra fibrilación auricular con respuesta ventricular rápida, signos de $\mathrm{MCH}-\mathrm{A}$ con ondas $\mathrm{T}$ negativas difusas. B) Radiografía de tórax con cardiomegalia a expensas de cavidades derechas y redistribución vascular. C) Ventana apical modificada donde se visualiza una insuficiencia tricuspídea severa y déficit de coaptación de los velos tricuspídeos. D) Imagen en ventana apical cuatro cámaras en las que se aprecia hipertrofia apical severa e insuficiencia tricuspídea severa. E) Registro del cateterismo derecho en el cual se objetiva el fenómeno de igualación de presiones de ambos ventrículos.

Sin embargo, la miectomía apical, llevada a cabo en centros especializados para aumentar la cavidad ventricular y el volumen latido, es una alternativa terapéutica en pacientes con $\mathrm{MCH}-\mathrm{A}$, de bajo gasto cardiaco y disfunción diastólica severa. En las diferentes series publicadas la supervivencia a largo plazo es superior al $80 \%^{5,6}$.

Se ha descrito que el $40 \%$ de los pacientes con $\mathrm{MCH}$ presentan IT siendo esta un predictor de síndrome cardiorrenal en ellos, tal como sucedió en nuestro $\mathrm{caso}^{7}$. La IT funcional, es decir, sin afectación orgánica de la válvula, habitualmente es debida a hipertensión pulmonar y/o cardiopatía izquierda. En una serie de pacientes con IT funcional severa y dilatación de anillo, el $9 \%$ no presentaba ninguna de estas condiciones y de ellos, en la mayoría la causa de la dilatación del anillo tricuspídeo fue la fibrilación auricular ${ }^{8}$. Como se ha puesto de manifiesto en varios trabajos, el remodelado auricular provocado por la fibrilación auricular puede provocar la dilatación del anillo tricuspídeo y originar la IT $^{9}$. 

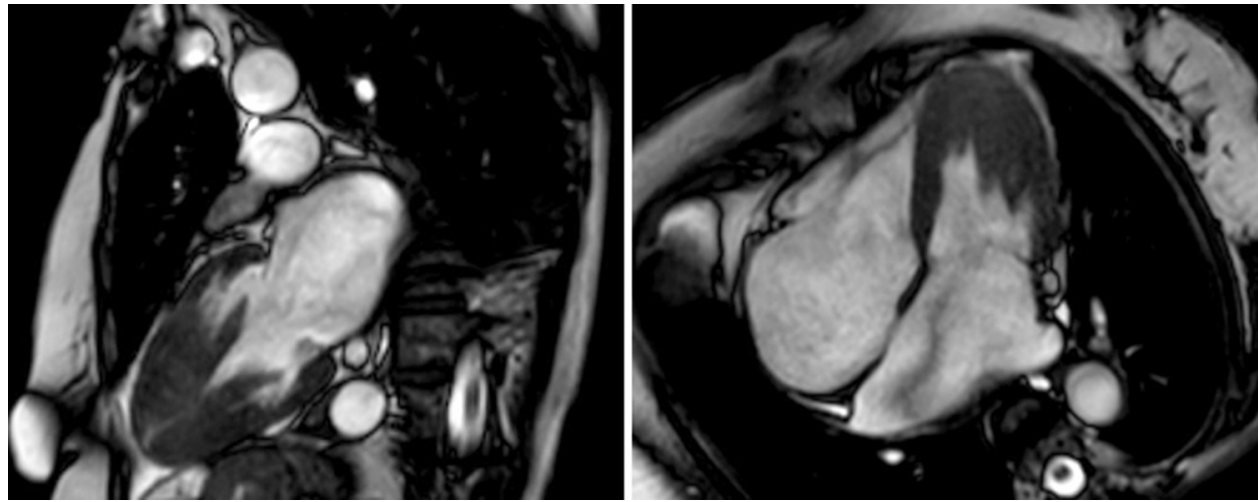

Figura 2 Imágenes de 2 y 4 cámaras de cardio-resonancia magnética en la cual se observa una hipertrofia severa apical del ventrículo izquierdo, grosor parietal del ventrículo derecho respetado y dilatación severa de ambas aurículas.

La caracterización adecuada del mecanismo de la IT y la exclusión de hipertensión pulmonar significativa son fundamentales a la hora de plantear la estrategia terapéutica en pacientes con cardiopatía izquierda para garantizar buen resultado de la anuloplastia. La presencia de IT severa se asocia a mal pronóstico con independencia de la cardiopatía subyacente y de la presión arterial pulmonar, y esto puede cambiar con el tratamiento quirúrgico de la misma realizando una adecuada selección ${ }^{10}$.

\section{Bibliografía}

1. Sakamoto T, Tei C, Murayama M, et al. Giant T wave inversion as a manifestation of asymmetrical apical hypertrophy (AAH) of the left ventricle: Echocardiographic and ultrasono-cardiotomographic study. Jpn Heart J. 1976;17: 611-29.

2. Kitaoka H, Doi Y, Casey SA, et al. Comparison of prevalence of apical hypertrophic cardiomyopathy in Japan and the United States. Am J Cardiol. 2003;92:1183-6.

3. Eriksson M, Sonnenberg B, Woo A, et al. Long-term outcome in patients with apical hypertrophic cardiomyopathy. J Am Coll Cardiol. 2002;39:638-45.

4. Arad M, Penas-Lado M, Monserrat L, et al. Gene mutations in apical hypertrophic cardiomyopathy. Circulation. 2005;112:2805-11.

5. Schaff H, Brown L, Dearani J, et al. Apical myectomy: A new surgical technique for management of severely symptomatic patients with apical hypertrophic cardiomyopathy. J Thorac Cardiovasc Surg. 2010;139:634-40.

6. Kunkala M, Schaff H, Nishimura R, et al. Transapical approach to myectomy for midventricular obstruction in hypertrophic cardiomyopathy. Ann Thorac Surg. 2013;96: 564-70.

7. Wang $X$, Peng J, Xie $X$, et al. Tricuspid regurgitation predicts cardiorenal syndrome in patients with hypertrophic cardiomyopathy. Int J Cardiol. 2015;197:83-4.
8. Mutlak D, Lessick J, Reisner SA, et al. Echocardiography-based spectrum of severe tricuspid regurgitation: The frequency of apparently idiopathic tricuspid regurgitation. J Am Soc Echocardiogr. 2007; 20:405-8.

9. Mutlak D, Aronson D, Lessick J, et al. Functional tricuspid regurgitation in patients with pulmonary hypertension: Is pulmonary artery pressure the only determinant of regurgitation severity? Chest. 2009;135:115-21.

10. Nath J, Foster E, Heidenreich PA. Impact of tricuspid regurgitation on long-term survival. J Am Coll Cardiol. 2004;43: 405-9.

Jesús Piqueras-Flores ${ }^{a, *}$, Alfonso Jurado-Román ${ }^{\mathrm{b}}$, Pedro Pérez-Díaz ${ }^{a}$, Ramón Maseda-Uriza ${ }^{a}$, Germán Hernández-Herra ${ }^{a}$, M. Ángeles Pérez-Martíneza ${ }^{a}$, María Thiscal López-Lluva ${ }^{\mathrm{b}}$, Juan Antonio Requena-lbáñez ${ }^{a}$, Raquel Frías-García ${ }^{a}$, Ignacio Sánchez-Pérez ${ }^{\mathrm{b}}$ y Fernando Lozano-Ruiz-Poveda ${ }^{\mathrm{b}}$

a Servicio de Cardiología, Hospital General Universitario de Ciudad Real, Ciudad Real, España

b Sección de Hemodinámica y Cardiología Intervencionista, Hospital General Universitario de Ciudad Real, Ciudad Real, España

* Autor para correspondencia. Servicio de Cardiología, Hospital General Universitario de Ciudad Real, C/ Obispo Torija, s/n, 13005 Ciudad Real, España. Teléfono: +637272079, Ext.: 79788, Fax: +926270800 .

Correo electrónico: jesus.piqueras.flores@gmail.com (J. Piqueras-Flores).

https://doi.org/10.1016/j.acmx.2017.10.001 1405-9940/

(c) 2017 Instituto Nacional de Cardiología Ignacio Chávez. Publicado por Masson Doyma México S.A. Este es un artículo Open Access bajo la licencia CC BY-NC-ND (http: / / creativecommons.org/ licenses/by-nc-nd/4.0/). 